\title{
Vaginal Practices and Associations with Barrier Methods and Gel Use Among Sub-Saharan African Women Enrolled in an HIV Prevention Trial
}

\author{
Ariane van der Straten $\cdot$ Helen Cheng • \\ Agnes Chidanyika • Guy De Bruyn • \\ Nancy Padian · the MIRA Team
}

Published online: 30 March 2010

(C) The Author(s) 2010. This article is published with open access at Springerlink.com

\begin{abstract}
Vaginal practices may interfere with the use and/or the effectiveness of female-initiated prevention methods. We investigated whether vaginal practices differed by randomization group in a phase III trial of the diaphragm with lubricant gel (MIRA) in Sub-Saharan Africa $(n=4925)$, and if they were associated with consistent use of study methods. At baseline, vaginal practices were commonly reported: vaginal washing $(82.77 \%)$, wiping $(56.47 \%)$ and insertion of dry or absorbent materials $(20.58 \%)$. All three practices decreased during the trial. However, women in the intervention group were significantly more likely to report washing or wiping during follow-up compared to those in the control group. Additionally, washing, wiping, and insertion, were all independently and inversely associated with consistent diaphragm and gel use and with condom use as well,
\end{abstract}

A. van der Straten $(\bowtie) \cdot H$. Cheng

Women's Global Health Imperative, RTI International, 114 Sansome Street, Suite 500, San Francisco, CA 94104, USA e-mail: ariane@rti.org

\section{A. van der Straten}

Department of Medicine, Center for AIDS Prevention Studies, University of California San Francisco, San Francisco, CA, USA

A. Chidanyika

UZ-UCSF Collaborative Research Programme,

University of Zimbabwe, Harare, Zimbabwe

A. Chidanyika $\cdot$ N. Padian

School of Public Health, University of California, Berkeley,

Berkeley, CA, USA

G. De Bruyn

Perinatal HIV Research Unit, University of the Witwatersrand, Johannesburg, South Africa regardless of study arm. A better understanding of the socio-cultural context in which these practices are embedded could improve educational strategies to address these potentially modifiable behaviors, and may benefit future HIV prevention interventions of vaginal methods.

Keywords Vaginal practices - HIV prevention trials . Female-initiated methods $\cdot$ Condoms $\cdot$ Africa

\section{Introduction}

In Sub-Saharan Africa, vaginal practices are widespread with prevalence reportedly ranging from 6 to $98 \%$ [1]. They are conducted for a variety of reasons including, but not limited to, genital hygiene, sexuality (sex preparation or sexual enhancement), and vaginal health/treatment of genital symptoms [2-4]. Women's genital hygiene is highly valued and there are clear norms regarding lubrication during sex, vaginal cleanliness and related vaginal practices. Specifically, the natural state of the vagina is expected to be dry and tight, with the interrelated positive sensations of cleanliness and warmth/heat generated by friction during sex. Consequently, women in this area are concerned with excessive wetness or lubrication, which may indicate disease, "dirt" in the vagina or a "loose" sexuality [4-7]. To maintain the desired vaginal state, a range of practices are used, including intravaginal cleansing and insertion of substances [4]. The most common substances used during vaginal practices are water, soap, commercial detergents, lemon or vinegar as well as traditional preparations with herbs or other dry materials such as cloth, paper or cotton wool $[8,9]$.

Given the high prevalence of vaginal practices in SubSaharan Africa, it is essential to understand whether these 
practices are associated and possibly interfere with the use of chemical or physical barrier methods that are currently being evaluated for their effectiveness in preventing HIV infection [3]. Female-initiated methods, such as microbicides and diaphragms, require vaginal insertion of a device or application of gel through a delivery system (single dose or reusable applicator, ring, diaphragm or other cervical barriers) [10-12]. While experience with vaginal practices may facilitate ease and comfort with inserting or removing these investigational vaginal methods, such habits, particularly if they occur for sex preparation and around the time of sex, could interfere with willingness to use and correct use of these methods (especially if such methods are coitally dependent), as well as their biological efficacy. The protocols for HIV prevention trials of female-initiated methods have often requested that participants abstain from vaginal practices, but it is not clear whether these guidelines have been followed [7, 13, 14].

To examine the relationship between vaginal practices and provision and use of female-initiated methods, we analyzed data from the MIRA study, an open-label multisite randomized controlled trial to assess the effectiveness of the diaphragm used with lubricant gel for the prevention of HIV/STI acquisition in HIV-negative women recruited from the general population in Southern Africa [15]. We previously reported that the diaphragm and gel had high acceptability among participants [16]. However, diaphragm adherence was sub-optimal and condom use was differential between study arms. [17, 18]. Continuing our examinations of factors influencing study methods use in MIRA, here, we investigated whether vaginal practices influenced use, whether practices changed during the trial, and if they differed between women randomized to the intervention (who received a diaphragm, vaginal gel and condoms), as compared to those in the control arm (who received male condoms only).

\section{Methods}

The MIRA trial was conducted in Harare, Zimbabwe, Durban and Johannesburg, South Africa, between 2003 and 2006 and included a total of 5,039 participants. Retention rate was high (93\%) and was similar between study randomization groups [15]. Women were followed quarterly for 12-24 months, depending on their calendar date of enrolment, with a median of 21 months follow-up or 7 quarterly visits (range 0-8 quarterly visits). The study protocol was reviewed and approved by all participating institutions' ethical review boards (www.ClinicalTrials.gov, NCT00121459); detailed trial procedures and primary results have been published elsewhere [15].
MIRA Trial Participants

Women were recruited from the general community and health facilities that provided services to women. Key eligibility criteria included being: sexually active; between 18 and 49 years; HIV negative; STI negative for cervical and vaginal STIs (or treatment thereof); with a healthy cervix, able to demonstrate the ability to correctly insert the diaphragm prior to randomization, and able to provide informed consent for participation.

\section{Study Procedures}

At the enrolment visit, all women received diaphragm education and demonstration using a pelvic model, and were fitted with a diaphragm by a trained clinician and practiced diaphragm insertion at the clinic as described previously [17]. Women completed a baseline Audio Computer Assisted Self-interviewing (ACASI) questionnaire (in local language) on: demographics, sexual behavior, vaginal practices, as well as current and previous use of the diaphragm, gel, and condoms. Eligible consenting women were then randomly assigned to intervention or control groups [15]. Because the intervention was not blinded, treatment assignment was known to the participant and clinical team (but not to the investigators and analysis team). At each quarterly follow-up visit, women completed an ACASI, and received: a clinical exam if indicated, product adherence and risk reduction counseling and a resupply of male condoms, as well as study gel (in the intervention group only). Use of effective contraception was encouraged and hormonal contraceptives were provided at no cost to all participants.

\section{Intervention and Product Counseling}

In the intervention group, women received a latex diaphragm (All_Flex Arcing Spring diaphragm: OrthoMcNeil Pharmaceutical, Raritan, NJ), a supply of noncontraceptive lubricant gel (Replens ${ }^{\circledR}$, Lil Drug Store Products, Cedar Rapids, IA) and male condoms. Women randomized to the control group received male condoms only. Participants in both groups received a comprehensive HIV prevention package including HIV/STI pre-and posttest counseling, treatment of curable laboratory-diagnosed STIs, condom promotion, and intensive risk reduction counseling.

Women in the intervention group were counseled to insert the diaphragm into the vagina any time convenient to them prior sexual intercourse, and to leave it in for $6 \mathrm{~h}$ post coitus. We counseled women to use the diaphragm with the lubricant gel by emptying an applicator of gel $(2.5 \mathrm{~g})$ into the dome of the diaphragm and spreading it onto the rim 
before application; additionally, women were asked to insert another applicator of gel in the vagina before each act of vaginal sex. At each visit, women were provided with a 3-month supply of the gel and could return to the clinic for more if desired. Because the effect of the diaphragm on HIV/STI prevention was unknown, all women were counseled to use condoms for every sexual act. The diaphragm is approved for contraception only when used with a spermicide [19], so participants were told not to use the diaphragm and lubricant gel for contraception. As part of study counseling, all participants were also discouraged from continuing any vaginal practices during the trial. Women were advised that they could only wash outergenitalia after sex with water or water and soap. Furthermore, women in the intervention group were specifically instructed not to clean the vagina or insert other vaginal products after they had inserted the diaphragm and gel, and before sex took place. Women, who nevertheless chose to continue vaginal practices, were encouraged to report and document them at follow-up visits.

\section{Measures}

Vaginal practices were assessed by ACASI at enrolment (and at quarterly follow-up visits) by asking participants the following three questions (five response categories: never to once per day or more, dichotomized for this analysis into Ever versus Never): Washing: "During the last 3 months (since your last computerized interview), how often did you wash inside your vagina?", Wiping: "During the last 3 months (since your last computerized interview), how often did you wipe, using dry cloth, paper, or cotton wool, inside your vagina?", Inserting: "During the last 3 months (since your last computerized interview), how often did you insert paper, tampon, cloth, or cotton wool into your vagina and leave it in for several hours or all day? (This does NOT include when you may have been menstruating.)"

Additionally, using ACASI, we also assessed the specific products used during these practices by women at two time points: baseline, and their last study visit. Women reported which specific product(s) they had used from the following list: water (only), soap and water, and "other products" which combines reported use of vinegar, commercial lubricant (such as KY jelly, Vaseline, or other), douche, natural agents (such as herbs, pulverized rock, mud, lemon, lemon juice, or other), commercial cleaning product (dettol, betadine, detergent, disinfectant, potassium permanganate, or other), or unspecified other products.

Study methods use: Among sexually active participants, we considered the following measures of study methods use assessed by ACASI at every quarterly visit: "Since your last visit, how often have you used a diaphragm when having sex?" (never, $\leq 50 \%,>50 \%$, always). Women were asked the same questions about gel use and about male condom use. For each time interval between two quarterly visits, women who reported always using the diaphragm and always using gel since their last visit, were classified as "consistent diaphragm and gel users" for that time interval; our primary outcome measure. We also created a dichotomous measure of consistent condom use ("always" used male condoms versus less than always) for each time interval.

We examined the following baseline variables (gathered through in-person interview or ACASI) as potential confounders in the multivariable models (described below). These were selected a priori, based on the literature and from prior published analyses of MIRA [15, 17, 20]: study site, age, education, cohabitation, marital status; having a positive test for any curable STI (Chlamydia, gonorrhea, trichomoniasis, syphilis), indicator variables both for participant's high-risk behavior and for having a high-risk partner (see Table 1 for definitions), and randomization group (for the full sample analyses).

\section{Statistical Analyses}

Our final analytic sample included 4,925 (97.73\%) of the total MIRA participants, who had at least one follow-up visit and responded to the quarterly vaginal practice questions of washing, wiping and insertion. Because of variations in vaginal practices and in consistent method use by study sites, all analyses described below were controlled for study site.

\section{Vaginal Practices Trends}

Preliminary analyses focused on tabulation of observed frequencies of various vaginal practices at each visit, overall, and by study randomization group. All comparisons were performed using GEE logistic regression to model a repeatedly measured binary outcome of whether a vaginal practice was reported by the participant. We compared specific vaginal practices at baseline and followup between the two study groups. We assessed change in vaginal practices over the follow-up period and included a visit by study group interaction term in these models to determine whether change in vaginal practices over the follow-up period differed by study group.

\section{Relationship Between Vaginal Practices and Use of Study Methods}

The associations between specific vaginal practices (a) at baseline and (b) at follow-up and our main method use outcome (consistent use of diaphragm/gel during follow- 
Table 1 Baseline socio-demographic characteristics, reproductive history and sexual behavior of MIRA participants

\begin{tabular}{|c|c|c|}
\hline Category & Total $(n=4925)$ & Total $(\%)$ \\
\hline \multicolumn{3}{|l|}{ Age (\#) } \\
\hline 24-year-old or younger & 1878 & 38.14 \\
\hline 25-34-year-old & 1936 & 39.32 \\
\hline 35-year-old or older & 1110 & 22.54 \\
\hline At least high school education ${ }^{a}$ & 2165 & 43.99 \\
\hline One lifetime partner (versus 2-30) & 2520 & 51.17 \\
\hline Married & 2917 & 59.23 \\
\hline Living together & 3342 & 67.86 \\
\hline \multicolumn{3}{|l|}{ Coital frequency (per week) } \\
\hline Three times or fewer & 3220 & 65.38 \\
\hline$>3$ times & 1705 & 34.62 \\
\hline Exchanged sex in past 3 months & 381 & 7.74 \\
\hline Tested positive for $\operatorname{STI}(\mathrm{s})^{\mathrm{b}}$ & 564 & 11.45 \\
\hline High behavior risk (at least one indicator versus none) ${ }^{\mathrm{c}}$ & 1406 & 28.55 \\
\hline High partner risk (at least one indicator vs. none)d,e & 3368 & 68.40 \\
\hline Ever used a diaphragm & 3 & 0.06 \\
\hline \multicolumn{3}{|l|}{ Frequency of condom use in past 3 months (enrollment) } \\
\hline Never & 1463 & 29.71 \\
\hline Sometimes & 1926 & 39.11 \\
\hline Always & 1536 & 31.19 \\
\hline \multicolumn{3}{|l|}{ Current contraceptive use (screening) } \\
\hline Long term ${ }^{\mathrm{f}}$ & 297 & 6.07 \\
\hline Injectable hormones & 1217 & 24.71 \\
\hline Pill $^{\mathrm{g}}$ & 1786 & 36.26 \\
\hline Barrier $^{\mathrm{h}}$ & 997 & 20.24 \\
\hline Other/none & 628 & 12.75 \\
\hline \multicolumn{3}{|l|}{ Study site } \\
\hline Harare & 2450 & 49.75 \\
\hline Durban & 1471 & 29.87 \\
\hline Johannesburg & 1004 & 20.39 \\
\hline
\end{tabular}

a 3 observations missing

b At least one positive test for CT, GC, TV or Syphilis at screening or enrollment

${ }^{c}$ Indicators include: any exchange of sex for money/food/drugs/shelter, two or more sexual partners within last 3 months, ever had vaginal sex under influence of drugs/alcohol in last 3 months, ever used needle for injectable drug use, ever had anal sex

${ }^{d}$ Indicators include: having any sexual partners test positive for HIV, suspect or know that regular partner had other sex partners in the last 3 months, ever had vaginal sex when partner was under influence of drugs/alcohol in last 3 months, regular partner was away from home $\geq 1$ months. Contraceptive methods are hierarchically coded by most effective methods

e 1 observation missing

${ }^{\mathrm{f}}$ Long term methods include tubal ligation, vasectomy, IUD, and implants such as Jadelle \& Norplant

g Pill methods include combined oral contraceptive and progesterone only pills

${ }^{\mathrm{h}}$ Barrier methods include male or female condoms

up) were examined within intervention group participants in univariate and multivariate models, using GEE logistic regression. We first examined separately each vaginal practice assessed either at baseline or at follow-up, for its association with the outcome. Although at baseline, the three vaginal practices were moderately correlated with each other (assessed by Pearson correlation coefficients, data not shown), there was no co linearity between them (assessed by the Variation Inflation Factor (VIF) values of approximately 1). Similarly, VIF values suggested no co linearity among the three vaginal practices at follow-up. Consequently, all three baseline and time-dependent exposure variables of washing, wiping and insertion were entered in one multivariate model. Next, potential baseline 
confounders of the association between vaginal practices and consistent use of study methods were examined. We selected variables that were significantly associated at the univariate level with the outcome. The final multivariate models only included predictors of interests and confounders that were significant at the $P<0.05$ level. Two hundred ninety-nine women HIV-seroconverted during the trial, and they were censored after their seroconversion visit because we hypothesized that this event would change their vaginal practices, sexual behavior and study method use.

Identical steps were used for the analyses of vaginal practices and consistent condom use during follow-up. In this case, we combined the two study groups (intervention and control) and adjusted for study group (and study site) as group assignment was not found to be an effect modifier of the vaginal practice and condom use relationship. All analyses were conducted using SAS version 9.1 (SAS Institute, Inc., Cary, NC, USA).

\section{Results}

\section{Study Sample Description and Vaginal Practices}

Our study included 4,925 MIRA participants with the following baseline characteristics (see Table 1): over onethird were 18-24-year-old; over half had only one lifetime partner, 59\% were married and $44 \%$ had at least a high school education. Half the sample was from Harare, $30 \%$ from Durban and $20 \%$ from Johannesburg. While $31 \%$ of women reported "always" using condoms in the past 3 months at their enrolment visit, only three women reported ever using a diaphragm.

As shown in Table 2, vaginal practices were prevalent: at baseline, most women (82.8\%) reported washing intravaginally in the previous 3 months $(61.4 \%$ reported this practice once a day or more). A subset of participants was asked during the first year of the trial what they used to wash their vagina. Of the 1,825 women who were asked this question, $72.9 \%$ reported using their finger, $27.8 \%$ reported using cloth and $4.7 \%$ other types of applicators (data not shown in table). Wiping intravaginally in the previous 3 months was reported by $56.5 \%$ of women (40.6\% reported wiping once a day or more) and $20.6 \%$ reported inserting dry or absorbent materials in their vagina (e.g. paper, tampon, cloth, or cotton wool) and leaving it for several hours in the previous 3 months (13.0\% reported insertion once a day or more). Few women (12.6\%) reported no practices, $31.8 \%$ reported one practice, and $56.6 \%$ reported $\geq 2$ practices. At baseline, there were no differences by randomization group in the three vaginal practices examined: washing, wiping, and insertion (Table 2). However, there were significant differences in the baseline prevalence of practices among the three study sites (data not shown in tables): washing was highest in Johannesburg (86.7 vs. $77.9 \%$ in Durban and $84.1 \%$ in Harare, Chi-square $P<0.0001)$, Zimbabwean women reported more frequent wiping (68.0 vs. $40.9 \%$ in Durban and $51.3 \%$ in Johannesburg, Chi-square $P<0.0001$ ) and insertion (25.6 vs. $12.9 \%$ in Durban and $19.4 \%$ in Johannesburg, Chi-square $P<0.0001$ ).

Specific product use was reported by more than half the baseline sample, and was balanced between randomization groups as well (Table 2). Use of soap and water was reported by a third of women, $29.2 \%$ used water alone, and only about $5 \%$ reported use of any other products. Durban reported the highest frequency of using products intravaginally (64.6 vs. $55.8 \%$ in Johannesburg and $44.9 \%$ in Harare, Chi-square $P<0.0001)$. Given that water or water and soap were mentioned most frequently, expectedly, specific product use was most correlated with washing $(r=0.20, P<0.0001)$ and less so with wiping $(r=0.06$, $P<0.0001)$ and insertion $(r=0.07, P<0.0001)$.

\section{Vaginal Practices During Follow-Up and by Study} Group

As shown in Table 2, during follow-up, there was a modest but significantly greater likelihood of reporting washing (AOR 1.35; 95\% CI 1.22-1.50, Chi-square $P<0.0001$ ), wiping (AOR 1.14; 95\% CI 1.05-1.24, Chi-square $P=0.002$ ) or use of soap and water (AOR 1.23 ; 95\% CI 1.07-1.41, Chi-square $P=0.003$ ) among women in the intervention group compared to those in the control group. There was no difference by study group in insertion practices or in use of water (alone) or other products in the vagina.

We further explored the change in vaginal practices over time: all three decreased with time in the study and the time effect was significant (Chi-square $P<0.0001$ ) for each vaginal practice overall, and within each study group (see Fig. 1a, b). Furthermore, as shown by the study group by visit interaction term (Fig. 1b), the rate of decrease over time in vaginal washing was significantly less in the intervention group compared with the control group (Chi-square $P=0.0004$ ). The rate of decrease for wiping was marginally less in the intervention group compared with the control group (Chi-square $P=0.05$ ). The study group effect was not observed for vaginal insertion.

\section{Vaginal Practices and Use of Study Methods During Follow-Up}

Of the total 27,469 persons-visits during the follow-up period, 25,905 (94.3\%) were intervals where women were sexually active, and thus, were included in the examination of the association between vaginal practices and consistent 
Table 2 Vaginal practices and product used (at baseline and follow-up) among MIRA participants, overall and by study group

\begin{tabular}{|c|c|c|c|c|c|c|c|}
\hline \multirow[t]{2}{*}{ Baseline } & \multicolumn{2}{|l|}{ Overall } & \multicolumn{2}{|c|}{ Intervention group } & \multicolumn{2}{|c|}{ Control group } & \multirow{2}{*}{$\begin{array}{l}\text { Chi-square } \\
P \text { value }\end{array}$} \\
\hline & $n=4925$ & $\%$ & $n=2466$ & $\%$ & $n=2459$ & $\%$ & \\
\hline Intravaginal washing & 4077 & 82.78 & 2053 & 83.25 & 2024 & 82.31 & ns \\
\hline Intravaginal wiping & 2782 & 56.49 & 1417 & 57.46 & 1365 & 55.51 & ns \\
\hline Intravaginal insertion & 1013 & 20.57 & 516 & 20.92 & 497 & 20.21 & ns \\
\hline Used product in vagina & 2608 & 52.95 & 1305 & 52.92 & 1303 & 52.99 & ns \\
\hline Water only & 1439 & 29.22 & 715 & 28.99 & 724 & 29.44 & $\mathrm{~ns}$ \\
\hline Soap and water & 1625 & 32.99 & 816 & 33.09 & 809 & 32.90 & $\mathrm{~ns}$ \\
\hline Other products & 269 & 5.46 & 138 & 5.60 & 131 & 5.33 & ns \\
\hline
\end{tabular}

Follow-up

\begin{tabular}{lllllllll}
\hline persons-visits $(\mathrm{pv})$ & $\begin{array}{l}n=27469 \\
\mathrm{pv}\end{array}$ & $\% \mathrm{pv}$ & $\begin{array}{l}n=13575 \\
\mathrm{pv}\end{array}$ & $\% \mathrm{pv}$ & $\begin{array}{l}n=13894 \\
\mathrm{pv}\end{array}$ & $\begin{array}{c}\% \text { pv } \\
\text { OR (95\%CI) }\end{array}$ & $\begin{array}{c}\text { Chi-square } \\
P \text {-value }\end{array}$ \\
\hline Intravaginal washing & 20821 & 75.8 & 10648 & 78.44 & 10173 & 73.22 & $1.35(1.22-1.50)$ & $<0.0001$ \\
Intravaginal wiping & 11247 & 40.94 & 5772 & 42.52 & 5475 & 39.41 & $1.14(1.05-1.24)$ & 0.0019 \\
Intravaginal insertion & 3613 & 13.15 & 1708 & 12.58 & 1905 & 13.71 & $0.93(0.82-1.04)$ & $\mathrm{ns}$
\end{tabular}

\begin{tabular}{lcccccccc}
\hline Women's last visit $^{\mathrm{b}}$ & $n=4711$ & $\%$ & $n=2353$ & $\%$ & $n=2358$ & $\%$ & \multicolumn{2}{c}{ OR (95\%CI) } \\
\hline Used product in vagina & 2192 & 46.53 & 1090 & 46.32 & 1102 & 46.73 & $0.98(0.88-1.1)$ & $\mathrm{ns}$ \\
Water only & 1220 & 25.9 & 620 & 26.35 & 600 & 25.45 & $1.05(0.92-1.2)$ & $\mathrm{ns}$ \\
Soap and water & 1277 & 27.11 & 679 & 28.86 & 598 & 25.36 & $1.23(1.07-1.41)$ & 0.003 \\
Other products & 198 & 4.2 & 95 & 4.04 & 103 & 4.37 & $0.92(0.69-1.23)$ & $\mathrm{ns}$ \\
\hline
\end{tabular}

All three vaginal practices and use of product in the vagina were asked for the past 3 months (at baseline) or since last quarterly interview (at follow-up visit)

Other products included at baseline: commercial cleaning product $(n=95 ; 1.9 \%)$; lubricants $(n=57 ; 1.2 \%)$; natural agents $(n=46 ; 0.9 \%)$; vinegar $(n=43 ; 0.9 \%)$, douche $(n=18 ; 0.4 \%)$, and other unspecified products $(n=46 ; 0.9 \%)$

Other products included at last visit: commercial cleaning product $(n=46 ; 0.9 \%)$; lubricants $(n=36 ; 0.8 \%)$; natural agents $(n=28 ; 0.6 \%)$; vinegar $(n=52 ; 1.1 \%)$, douche $(n=23 ; 0.5 \%)$, and other unspecified products $(n=40 ; 0.9 \%)$

$O R$ odds ratio intervention versus control arm, controlling for site, $C I$ confidence interval, $n s$ non-significant, $p v$ persons-visits

${ }^{a}$ GEE

b This question was assessed at participant's exit visit or last available study visit, in a subset of 4,711 women

${ }^{c}$ logisitic regression

use of study methods. The association between vaginal practices and consistent use of diaphragm/gel was examined among women in the intervention arm only $(n=2438)$, for a total of 12,853 persons-visits. During their time in the study, women reported always using diaphragms in the previous 3 months at $58.1 \%$ of all follow-up visits (with visit-specific proportions ranging from 54.5 to $61.8 \%$ ) and reported always using gel at $59.5 \%$ of all follow-up visits (with visit-specific proportions ranging from 56.0 to $62.2 \%$ ). Reports of consistent diaphragm and gel use (always using both in the previous 3 months) were reported at $52.2 \%$ of follow-up visits (with visit-specific proportions ranging from 48.1 to $55.0 \%$ ). As shown in Table 3, baseline vaginal practices were not associated with consistent use of the diaphragm and gel during followup. However, in multivariable analysis, follow-up reports of washing (AOR 0.88; 95\% CI 0.79-0.98), wiping (AOR
0.90 ; 95\% CI 0.83-0.97), and insertion (AOR 0.83; 95\% CI 0.74-0.93), were all independently and inversely associated with consistent use of the diaphragm and gel during the same time intervals, after controlling for significant confounding baseline variables (study site, age, educational status, women's behavioral risk, high risk partner).

Similarly, we examined the association between vaginal practices and consistent male condom use in the complete study sample. During their time in the study, women reported always using condoms in the previous 3 months at $60.7 \%$ of all follow-up visits (with visit-specific proportions ranging from 59.8 to $61.9 \%$ ); $59.08 \%$ (range $58.1-$ $60.6 \%$ ) in the intervention group and $62.3 \%$ (range 61.0 $3.2 \%$ ) in the control group (OR $0.87 ; 95 \%$ CI $0.80-0.95$ ). As shown in Table 4, baseline washing, wiping and specific vaginal product use, were significantly associated with a lower likelihood of consistent use of male condoms 


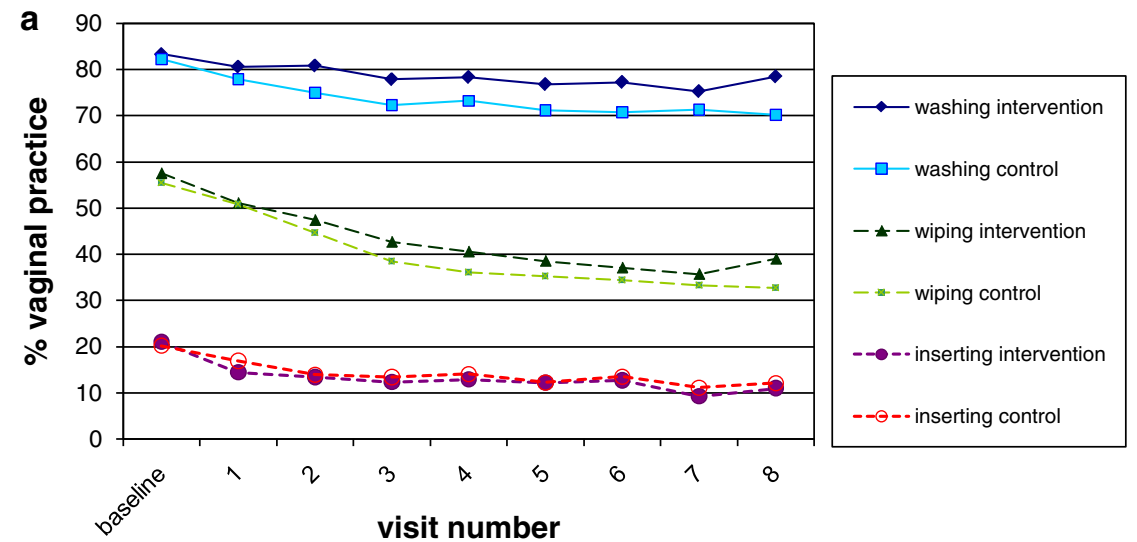

\begin{tabular}{|c|c|c|c|}
\hline \multicolumn{2}{|c|}{ Study Arm-Specific } & \multicolumn{2}{|c|}{$\begin{array}{l}\text { Study Group by Visit } \\
\text { Interaction }\end{array}$} \\
\hline $\begin{array}{c}\text { Odds Ratio } \\
(95 \% \mathrm{Cl})\end{array}$ & $\begin{array}{c}\text { Chi-square } \\
\text { p-value }\end{array}$ & $\begin{array}{c}\text { Odds Ratio } \\
(95 \% \mathrm{Cl})\end{array}$ & $\begin{array}{c}\text { Chi-square } \\
\text { p-value }\end{array}$ \\
\hline $.95(.94-.97)$ & $p<.0001$ & \multirow{3}{*}{$.97(.95-.99)$} & \multirow{3}{*}{0.0004} \\
\hline & & & \\
\hline $.92(.91-.94)$ & $\mathrm{p}<.0001$ & & \\
\hline $.89(.88-.90)$ & $p<.0001$ & \multirow{3}{*}{$.98(.97-1.0)$} & \multirow{3}{*}{0.05} \\
\hline & & & \\
\hline $.87(.86-.89)$ & $p<.0001$ & & \\
\hline $.92(.91-.94)$ & $p<.0001$ & \multirow{3}{*}{$1.01(.98-1.03)$} & \multirow{3}{*}{0.66} \\
\hline & & & \\
\hline $.93(.91-.94)$ & $p<.0001$ & & \\
\hline
\end{tabular}

Fig. 1 a Percent women reporting specific vaginal practices over time and by study group proportion of women within each study group, reporting "ever" engaging in a specified vaginal practice at every quarterly visit. Visit number: Baseline $=1,3$-month follow-up visit $=3$, 6-month visit $=4$, 9-month visit $=5$, etc. $\mathbf{b}$ Linear trend test for specific vaginal practices, by study group study arm-specific: within-group linear trend tests for the three vaginal practices,

controlling for repeated measures (with GEE) and study site. Odds ratio indicates odds of "ever" engaging in specified vaginal practice at one visit compared to previous visit. Vaginal practices (washing, wiping and insertion) were measured at baseline and every quarterly visit. Study Arm by visit interaction: for each specified vaginal practice, odds ratio of study arm by visit interaction term (with intervention group as reference category). $95 \%$ CI $95 \%$ confidence interval

Table 3 Univariate and multivariate models of the association between specific vaginal practices at baseline and follow-up, and consistent diaphragm/gel use among MIRA Intervention Group participants (2,438 participants; 12,853 persons-visits)

\begin{tabular}{|c|c|c|c|c|c|c|c|c|}
\hline \multirow[t]{2}{*}{ Vaginal practices } & \multicolumn{4}{|c|}{ Univariate analyses } & \multicolumn{4}{|c|}{ Multivariate analyses } \\
\hline & $\begin{array}{l}\text { OR } \\
\text { estimate }\end{array}$ & $\begin{array}{l}\text { Lower } \\
95 \% \text { CI }\end{array}$ & $\begin{array}{l}\text { Upper } \\
95 \% \text { CI }\end{array}$ & $\begin{array}{l}\text { Chi-square } \\
P \text {-value }\end{array}$ & $\begin{array}{l}\text { AOR } \\
\text { estimate }\end{array}$ & $\begin{array}{l}\text { Lower } \\
95 \% \text { CI }\end{array}$ & $\begin{array}{l}\text { Upper } 95 \% \\
\text { CI }\end{array}$ & $\begin{array}{l}\text { Chi-square } \\
P \text {-value }\end{array}$ \\
\hline \multicolumn{9}{|l|}{ Baseline measures } \\
\hline Baseline intravaginal washing & 0.92 & 0.79 & 1.08 & ns & na & & & \\
\hline Baseline intravaginal wiping & 0.99 & 0.88 & 1.12 & ns & na & & & \\
\hline Baseline intravaginal insertion & 1.12 & 0.97 & 1.29 & ns & na & & & \\
\hline Baseline used product in vagina* & 0.91 & 0.81 & 1.03 & ns & na & & & \\
\hline \multicolumn{9}{|l|}{ Repeated-measures at follow up } \\
\hline Intravaginal washing at follow-up* & 0.83 & 0.76 & 0.92 & 0.0003 & 0.88 & 0.79 & 0.98 & 0.0147 \\
\hline Intravaginal wiping at follow-up ${ }^{a}$ & 0.86 & 0.80 & 0.93 & $<0.0001$ & 0.90 & 0.83 & 0.97 & 0.0083 \\
\hline Intravaginal insertion at follow-up ${ }^{a}$ & 0.80 & 0.72 & 0.90 & $<0.0001$ & 0.83 & 0.74 & 0.93 & 0.0014 \\
\hline
\end{tabular}

${ }^{a}$ In univariate analyses, washing, wiping and insertion at follow-up visits were controlled for at study site

Multivariate analyses controlled for baseline factors and potential confounders that remained significant at $P<0.05$ : study site, age, educational status, women's behavioral risk, high risk partner

$O R$ odds ratio, $A O R$ adjusted $\mathrm{OR}, C I$ confidence interval, $n a$ not applicable, $n s$ non significant

during follow-up. In multivariable analyses, only baseline use of vaginal products remained significant (AOR 0.90; 95\% CI 0.82-0.98). Additionally, follow-up reports of washing (AOR 0.88; 95\% CI 0.82-0.94), wiping (AOR $0.94 ; 95 \%$ CI 0.89-0.99), and insertion (AOR 0.89; 95\% CI $0.82-0.97$ ), were all independently and inversely associated with consistent use of male condoms during followup, after controlling for significant confounding baseline variables (study group, study site, age, marital and educational status, women's behavioral risk, high risk partner, cohabitation, positive curable STI at baseline).

\section{Discussion}

We report high prevalence of vaginal practices among women who participated in MIRA, a multisite HIV prevention trial of the diaphragm used with lubricant gel. Baseline prevalence was higher than in other studies conducted in similar geographical areas (29\% in South Africa [21]; 66\% in Zimbabwe and Uganda [22]). This may result from the use of different question types to assess vaginal practices; or from interview mode, as ACASI has been shown to increase selfreporting of these socially sensitive behaviors [23]. 
Table 4 Univariate and multivariate models of the association between specific vaginal practices at baseline and follow-up, and consistent condom use among MIRA participants (both study groups combined) (4874 participants, 25,905 persons-visits)

\begin{tabular}{|c|c|c|c|c|c|c|c|c|}
\hline \multirow[t]{2}{*}{ Vaginal practices } & \multicolumn{4}{|c|}{ Univariate analyses } & \multicolumn{4}{|c|}{ Multivariate analyses } \\
\hline & $\begin{array}{l}\text { OR } \\
\text { estimate }\end{array}$ & $\begin{array}{l}\text { Lower } \\
95 \% \text { CI }\end{array}$ & $\begin{array}{l}\text { Upper } \\
95 \% \text { CI }\end{array}$ & $\begin{array}{l}\text { Chi-square } \\
P \text {-value }\end{array}$ & $\begin{array}{l}\text { AOR } \\
\text { estimate }\end{array}$ & $\begin{array}{l}\text { Lower } \\
95 \% \text { CI }\end{array}$ & $\begin{array}{l}\text { Upper } \\
95 \% \text { CI }\end{array}$ & $\begin{array}{l}\text { Chi-square } \\
P \text {-value }\end{array}$ \\
\hline \multicolumn{9}{|l|}{ Baseline measures } \\
\hline Baseline intravaginal washing & 0.89 & 0.79 & 0.99 & 0.033 & na & & & \\
\hline Baseline intravaginal wiping & 0.84 & 0.77 & 0.91 & $<.0001$ & na & & & \\
\hline Baseline intravaginal insertion & 0.91 & 0.82 & 1.01 & ns & na & & & \\
\hline Baseline used product in vagina ${ }^{a}$ & 0.88 & 0.81 & 0.97 & 0.0064 & 0.90 & 0.82 & 0.98 & 0.021 \\
\hline \multicolumn{9}{|l|}{ Repeated-measures at follow up } \\
\hline Intravaginal washing at follow-up ${ }^{a}$ & 0.83 & 0.78 & 0.89 & $<.0001$ & 0.88 & 0.82 & 0.94 & 0.0002 \\
\hline Intravaginal wiping at follow-up ${ }^{a}$ & 0.89 & 0.85 & 0.94 & $<.0001$ & 0.94 & 0.89 & 0.99 & 0.0327 \\
\hline Intravaginal insertion at follow-up ${ }^{a}$ & 0.86 & 0.80 & 0.93 & 0.0002 & 0.89 & 0.82 & 0.97 & 0.0067 \\
\hline
\end{tabular}

${ }^{a}$ In univariate analyses, washing, wiping and insertion at follow-up visits were controlled for at study site

Multivariate analyses controlled for baseline factors and potential confounders that remained significant at $P<0.05$ : study group, study site, age, married, educational status, women's behavioral risk, high risk partner, cohabitation, postive curable STI at baseline

$O R$ odds ratio, $A O R$ adjusted $\mathrm{OR}, C I$ confidence interval, $n a$ not applicable, $n s$ non significant

Standardized counseling in MIRA discouraged vaginal practices or use of non-study vaginal products for all participants. It is thus plausible that these practices reportedly decreased during the trial because of the counseling women received or because of social desirability bias. Of note, washing decreased the least, while vaginal insertion decreased the most, suggesting that certain practices may be less amenable to change than others. Specifically, local norms about women's genital hygiene place great value on "vaginal cleanliness" and excessive vaginal secretion and/ or post-coital discharges are viewed as "dirty" [4, 5, 7, 24, 25]. Furthermore, keeping the vagina clean and removing undesirable discharge are not just motivated by hygiene but also by local views of sexuality, such as the expectation that women maintain their vagina in a "virgin-like state" by removing any evidence of prior sexual activity, to satisfy the sexual needs of the male partner and to ensure his fidelity [6]. Thus, daily vaginal washing as well as postcoital washing may be more likely to persist despite repeated counseling.

Women in the intervention group, who received diaphragm, gel and condoms, had a greater likelihood of reporting vaginal practices during follow-up compared to those in the control group, who received condoms only. This occurred despite the fact that overall, reported practices decreased over time in both groups. Manual insertion and removal of the diaphragm could have encouraged more frequent vaginal practices, such as finger cleansing. Also gel may add to the amount of post-coital discharge, and increase the likelihood of vaginal cleaning after sex. Alternatively, given that in MIRA, the study gel was generally liked and was believed to have "cleansing" properties [16] (Sahin-Hoduglugil, personal communication,
August 2009), participants may have used it as a cleansing tool. Further research should aim to better understand women's motivations for vaginal practices and to tease out these possible alternative explanations. Of note, local beliefs and perceptions about beneficial and cleansing properties of a potential microbicide were also reported in a study of South African sex workers [26] and in a large microbicide trial [27]. Thus, while studies have shown that some level of lubrication during sex is welcomed, and that lubricant and microbicide acceptability is generally high in settings where vaginal practices are prevalent [28$30]$, it is possible that gel use may sustain or increase certain vaginal practices. In contrast, use of male condoms should prevent any post-coital discharge, and hence, this may in part explain lower vaginal practices reported in the control group. Condoms were equally promoted in both groups, but reported use was lower in the intervention group [15].

Our findings also indicate that vaginal practices were associated with a lower likelihood of consistently using the investigational products (diaphragm and gel) among women in the intervention group, as well as a lower likelihood of consistently using condoms, overall. Although the effects were small, the associations were independently associated with all three vaginal practices examined, and cumulatively may add up to a substantial effect, specially, given that most women reported more than one type of practice. Only baseline use of vaginal products was independently associated with a lower likelihood of consistent condom use, suggesting a potential causal relationship. All other associations between vaginal practices and methods use were concurrently assessed during follow-up, and thus could be correlated without being causally related. Of note, 
small exploratory studies in Zimbabwe and Kenya reported that some vaginal practices may interfere with correct or consistent use of the diaphragm [31, 32]. We also found that vaginal wiping was associated with lower diaphragm insertion skills in this study population [33].

Several other qualitative studies have indicated that vaginal practices may interfere with use and effectiveness of condoms [6, 34, 35]. Use of condoms may conflict with sexual norms favoring the exchange of fluids and positive sensations of heat caused by "skin to skin" friction during sex. Also the effects of intravaginal agents used for tightening, warming or improving the sexual experience may not be felt across a physical barrier. For example, in a qualitative study in Zimbabwe, some women were reluctant to use condoms for fear of blocking the 'magic' of drying agents [5]. However this study is amongst the first to quantitatively show in a large sample of women that such associations exist.

There are several limitations to this study: first, we did not use standardized measures of vaginal practices nor did we have detailed measures, including when these vaginal practices took place in relation to sex or to use of study methods [4]. Measures of vaginal practices and of study method use were self-reported and thus, susceptible to social desirability bias. However, this would make women reluctant to report both vaginal practices and inconsistent study method use, decreasing the likelihood of finding an association between the two. Also, because we had no a priori reason to expect differential reports of vaginal practices by study arm, our analysis of differences in vaginal practices by randomization group should be quite robust.

There is an urgent need to establish which vaginal practices are harmful to women's health and which are not. Recently a Vaginal Practice Research Partnership (VPRP) was created to investigate evidence about the hypothesized causal pathway between women's vaginal practices and acquisition of HIV infection. The Partnership is conducting an individual patient data (IPD) meta-analysis of studies identified in a systematic review of the research literature, and preliminary data from nine cohort studies (including MIRA) suggest an increased risk of HIV associated with certain vaginal practices, particularly practices other than cleansing with water [36]. Another way vaginal practices could be harmful is if they interfere with HIV prevention practices. Here, we report an inverse relationship between vaginal practices and consistent use of HIV prevention methods evaluated in the MIRA trial. Although these are potentially modifiable behaviors, changing vaginal practices may not be straightforward given their cultural and social meanings. Still, by better understanding the motivations and needs behind these practices, it may be possible to educate women, and suggest healthy alternatives that can fulfill underlying motivations. For example, anecdotal information in Zimbabwe indicated that mint flavored condoms (who provided a refreshing and tingling sensation) could be substituted for vaginal insertion of Vicks, a mint-based commercial product [5]. In MIRA, participants and their partners were provided with standard and flavored condoms (i.e. mint, strawberry, banana, chocolate), and these flavoring additives appeared to make condom use more likeable (H. Cheng, personal communication, November 2009). Also most women reportedly "prepare for sex" by washing their genitalia daily [7]. Researchers could capitalize on this habit to suggest daily insertion of a microbicide to "prepare for sex", especially if its formulation induces vaginal sensations of cleanliness and freshness, as reported with some gels [27, 37, 38]. Finally, as discussed above, the perceived "cleansing" properties of investigational gels, could serve to encourage gel insertion and discourage early removal or coinsertion of other substances.

In summary, future studies should help distinguish "harmful" vaginal practices from "harmless" ones, and their potential effects on microbicides and other vaginal methods' acceptability, effectiveness and safety. Further research on the meaning and motivation of these practices could inform the development of improved educational strategies to address these modifiable behaviors in the context of HIV prevention interventions, to maximize consistent and correct vaginal method use and minimize potential interference by existing vaginal practices.

Acknowledgements We would like to thank the women who participated in this study. Special thanks to Drs. van der Wijgert and Martin Hilber for reviewing an earlier version of this manuscript, and to Katharine Rivett for editorial assistance. The MIRA trial was funded through a grant from the Bill and Melinda Gates Foundation (\#21082) and included the following collaborating organizations: University of California San Francisco, San Francisco, CA; Ibis Reproductive Health, Cambridge, MA; the UZ-UCSF collaborative research Programme, Harare, Zimbabwe; the Medical Research Council, Durban; and The University of Witswatersand, Johannesburg, South Africa. For A. van der Straten, H. Cheng, and N. Padian most work for this study was conducted at the University of California San Francisco, Department of Obstetrics, Gynecology and Reproductive Sciences.

Open Access This article is distributed under the terms of the Creative Commons Attribution Noncommercial License which permits any noncommercial use, distribution, and reproduction in any medium, provided the original author(s) and source are credited.

\section{References}

1. Meyer L, Kuhn L, Stein Z, et al. Vaginal practices, bacterial vaginosis, and women's susceptibility to HIV infection: epidemiological evidence and biological mechanisms. Lancet Infect Dis. 2005;5:786-94.

2. Brown JE, Brown RC. Traditional intravaginal practices and the heterosexual transmission of disease: a review. Sex Transm Dis. 2000;27(4):183-7. 
3. Hilber MA, Chersich MF, van de Wijgert J, et al. Vaginal practices, microbicides and HIV: what do we need to know? (Editorial). Sex Transm Inf. 2008;83(7):505-8.

4. Hilber AM, Hull T, Preston-Whyte E, et al. A cross cultural study of vaginal practices and sexuality: implications for sexual health. Soc Sci Med. 2010;70(3):392-400.

5. Civic D, Wilson D. Dry sex in Zimbabwe and implications for condom use. Soc Sci Med. 1996;42(1):91-8.

6. Scorgie F, Kunene B, et al. In search of sexual pleasure and fidelity: vaginal practices in KwaZulu-Natal, South Africa. Cult Health Sex. 2009;11(3):267-83.

7. Braunstein S, Wijgert JVD. Preferences and practices related to vaginal lubrication: implications for microbicide acceptability and clinical testing. J Women's Health. 2005;14(5):424-33.

8. Dallabetta G, Miotti P, Chiphangwi J, et al. Traditional vaginal agents: use and association with HIV infection in Malawain women. AIDS. 1995;9:293-7.

9. van de Wijgert JH, Mason PR, Gwanzura L, et al. Intravaginal practices, vaginal flora disturbances, and acquisition of sexually transmitted diseases in Zimbabwean women. J Infect Dis. 2000;181(2):587-94.

10. Romano J, Malcolm R, Garg S, et al. Microbicide delivery: formulation technologies and Strategies. Curr Opin HIV AIDS. 2008;3(5):558-66.

11. Ballag S, Brache V, Mauck C, et al. A Phase 1 study of the functional performance, safety, and acceptability of the BufferGel Duet. Contraception. 2008;77(2):130-7.

12. Ellertson C, Burns M. Re-examining the role of cervical barrier devices. Outlook. 2003;20(2):1-8.

13. Behets F, Van Damme K, Turner A, et al. Evidence-based planning of a randomized controlled trial on diaphragm use for prevention of sexually transmitted infections. Sex Transm Dis. 2008;35(3):238-42.

14. Behets F, Turner A, Van Damme K, et al. Vaginal microbicide and diaphragm use for sexually transmitted infection prevention: a randomized acceptability and feasibility study among high-risk women in Madagascar. Sex Transm Dis. 2008;35(9):818-26.

15. Padian NS, van der Straten A, Ramjee G, et al. Diaphragm and lubricant gel for prevention of HIV acquisition in southern African women: a randomised controlled trial. Lancet. 2007; 370(9583):251-61.

16. Montgomery ET, Cheng H, van der Straten A, et al. Acceptability and use of the diaphragm and Replens((R)) Lubricant Gel for HIV prevention in Southern Africa. AIDS Behav. 2009 [epub ahead of print].

17. van der Straten A, Shiboski S, Montgomery ET, et al. Patterns and predictors of adherence to diaphragm use in a Phase III trial in sub-Saharan Africa: a trajectory analysis. J Acquire Immune Defic Syndr. 2009;50(4):419-26.

18. van der Straten A, Cheng H, Moore J, et al. The use of the diaphragm instead of condoms in a phase III diaphragm trial. AIDS Behav. 2009;13(3):564-72.

19. Cook LA, Nanda K, Grimes DA, et al. Diaphragm versus diaphragm with spermicides for contraception. Coch Database Syst Rev. 2003, Issue 1. Art. No.: CD002031.

20. Ramjee G, van der Straten A, Chipato T, et al. The diaphragm and lubricant gel for prevention of cervical sexually transmitted infections: results of a randomized controlled trial. PLoS One. 2008;3(10):3488

21. Meyer L, Denny L, de Souza M, et al. Vaginal practices, HIV and other sexually transmitted diseases among South African women. Sex Transm Dis. 2004;31(3):174-9.
22. van de Wijgert $\mathrm{J}$, Morrison $\mathrm{C}$, Cornelisse $\mathrm{P}$, et al. Bacterial vaginosis and vaginal yeast, but not vaginal cleansing, increase HIV-1 acquisition in African women. J Acquir Immune Defic Syndr. 2008;48:203-10.

23. Mensch B, Hewett $P$, Abbot $S$, et al. Assessing the reporting of adherence and sexual activity in a simulated microbicide trial in South Africa: an interview mode experiment using a placebo gel. Presented at the International Conference for Microbicide, Delhi, India, 2008.

24. Buck J, Kang M, van der Straten A, et al. Barrier method preferences and perceptions among Zimbabwean women and their partners. AIDS Behav. 2005;9(4):415-22.

25. Okal J, Stadler J, et al. Secrecy, disclosure and accidental discovery: perspectives of diaphragm users in Mombasa, Kenya. Culture, Health \& Sexuality. Int J Res Interv Care. 2008;10(1): $13-26$.

26. Mantell JE, Morar NS, Myer L, Ramjee G. We have our protector: misperceptions of protection against HIV among participants in a microbicide efficacy trial. Am J Public Health. 2006; 96(6):1073-7.

27. Saethre EJ, Stadler J. Gelling medical knowledge: innovative pharmaceuticals, experience, and perceptions of efficacy. Anthropol Med. 2010;17(1):99-111.

28. Veldhuijzen N, Nyinawabega J, Umulisa M, et al. Preparing for microbicide trials in Rwanda: focus group discussions with Rwandan women and men. Cult Health Sex. 2006;8(5):395-406.

29. Woodsong C, Alleman P. Sexual pleasure, gender power and microbicide acceptability in Zimbabwe and Malawi. AIDS Educ Prev. 2008;20(2):171-87.

30. Bentley ME, Fullem AM, Tolley EE, et al. Acceptability of a microbicide among women and their partners in a 4-country phase I trial. Am J Public Health. 2004;94(7):1159-64.

31. Sharma A, Bukusi E, Posner S, et al. Sex preparation and diaphragm acceptability in sex work in Nairobi, Kenya. Sex Health. 2006;3(4):261-8.

32. van der Straten A. Intravaginal practices and diaphragm use in Zimbabwe (poster presentation). Paper presented at Microbicides Conference, Cape Town, South Africa, April 23-26, 2006.

33. Montgomery ET, Blanchard K, Cheng H, et al. Diaphragm and lubricant gel acceptance, skills and patterns of use among women in an effectiveness trial in Southern Africa. Eur J Contracept Reprod Health Care. 2009;14(6):410-9.

34. Ray S, Gumbo N, Mbvizo M. Local voices: what some Harare men say about preparation for sex. Reprod Health Matters. 1996;7:34-45.

35. Bagnol B, Mariano E. Vaginal practices: eroticism and implications for women's health and condom use in Mozambique. Cult Health Sex. 2008;10(6):573-85.

36. Chersich M, Martin Hilber A, Schmidlin K, et al. Association between intravaginal practices and HIV acquisition in women: individual patient data meta-analysis of cohort studies in subSaharan Africa (oral presentation). International AIDS Society (IAS) Conference, Cape Town, SA, 2009.

37. Bentley ME, Morrow KM, Fullen A, et al. Acceptability of a novel vaginal microbicide during a safety trial among low-risk women. Fam Plann Perspect. 2000;32(4):1-5.

38. Coggins $\mathrm{C}$, Blanchard K, Alvarez F, et al. Preliminary safety and acceptability of a carrageenan gel for possible use as a vaginal microbicide. Sex Transm Infect. 2000;76(6):480-3. 